\title{
Using ground reaction force to predict knee kinetic asymmetry following anterior cruciate ligament reconstruction
}

\author{
B. Dai ${ }^{1,4}$, R. J. Butler ${ }^{1,2,3}$, W. E. Garrett ${ }^{1,2}$, R. M. Queen ${ }^{1,2}$ \\ ${ }^{1}$ Michael W. Krzyzewski Human Performance Laboratory, Duke University, Durham, North Carolina, USA, ${ }^{2}$ Department of \\ Orthopaedic Surgery, Duke University Medical Center, Durham, North Carolina, USA, ${ }^{3}$ Community and Family Medicine, Division of \\ Doctor of Physical Therapy, Duke University, Durham, North Carolina, USA, ${ }^{4}$ Division of Kinesiology and Health, University of \\ Wyoming, Laramie, Wyoming, USA \\ Corresponding author: Robin M. Queen, PhD, 102 Finch Yeager Building - DUMC 3435, Durham, North Carolina 27710 , USA. \\ Tel: (919) 684-1853, Fax: (919) 681 7067, E-mail: robin.queen@duke.edu
}

Accepted for publication 1 August 2013

Asymmetries in sagittal plane knee kinetics have been identified as a risk factor for anterior cruciate ligament (ACL) re-injury. Clinical tools are needed to identify the asymmetries. This study examined the relationships between knee kinetic asymmetries and ground reaction force (GRF) asymmetries during athletic tasks in adolescent patients following ACL reconstruction (ACL-R). Kinematic and GRF data were collected during a stopjump task and a side-cutting task for 23 patients. Asymmetry indices between the surgical and non-surgical limbs were calculated for GRF and knee kinetic variables. For the stop-jump task, knee kinetics asymmetry indices were correlated with all GRF asymmetry indices
$(P<0.05)$, except for loading rate. Vertical GRF impulse asymmetry index predicted peak knee moment, average knee moment, and knee work $\left(R^{2} \geq 0.78, P<0.01\right)$ asymmetry indices. For the side-cutting tasks, knee kinetic asymmetry indices were correlated with the peak propulsion vertical GRF and vertical GRF impulse asymmetry indices $(P<\mathbf{0 . 0 5})$. Vertical GRF impulse asymmetry index predicted peak knee moment, average knee moment, and knee work $\left(R^{2} \geq 0.55, P<0.01\right)$ asymmetry indices. The vertical GRF asymmetries may be a viable surrogate for knee kinetic asymmetries and therefore may assist in optimizing rehabilitation outcomes and minimizing re-injury rates.
The anterior cruciate ligament (ACL) injury rate is approximately 1 in 3000 in the general population (Miyasaka et al., 1991; Granan et al., 2008). These injuries typically occur during jump landing, cutting, and pivoting tasks with a non-contact mechanism of injury (Boden et al., 2000). ACL reconstruction (ACL-R) is commonly performed for a patient who has sustained an ACL rupture. The aim of this surgical procedure is to restore knee stability and increase the chance to return to sports (Fink et al., 2001). However, ACL graft and contralateral ACL rupture rates are more than $10 \%$ in the general population (Salmon et al., 2005; Shelbourne et al., 2009a) and more than $20 \%$ in adolescent and young athletes who returned to the sports of soccer and basketball following ACL-R (Shelbourne et al., 2009b).

One neuromuscular control risk factor that has been identified for ACL re-injury was asymmetry in sagittal plane knee joint moment during landing (Paterno et al., 2010). However, significant limb kinetic asymmetries have been observed in ACL injured patients who were cleared to return to play (Orishimo et al., 2010). Orishimo et al. (2010) compared knee kinetics between surgical and non-surgical limbs during a single-leg hopping test in adult patients following ACL-R. The researchers showed that the average ratio (involved/noninvolved) of hopping distance was $93 \%$; however, the average ratio of peak knee extension moment was only $60 \%$. The findings suggest that the restoration of symmetry in common clinical testing (Kvist, 2004; Barber-Westin \& Noyes, 2011) does not guarantee knee kinetic symmetry during athletic tasks. Decreased knee moments on the surgical side suggest decreased utilization of the surgical knee to complete athletic tasks and thus supports the notion that the surgical knee has not fully recovered to a functional level. Failure to identify this asymmetry might misguide an athlete's readiness for return to activity following ACL reconstruction and thus increase the athlete's risk of a second ACL injury.

The most common way of identifying knee kinetic asymmetries during athletic tasks is by utilizing an inverse dynamics approach with motion capture and ground reaction force (GRF) measurements. However, the resource demands and high cost of performing this inverse dynamics limit its application in many medical settings (Myer et al., 2010). It is important to develop simple, low cost, and accurate measurements as clinically applicable tools to identify knee kinetic asymmetries. From the perspective of inverse dynamics, GRFs 


\section{Dai et al.}

are determinant components in calculating knee kinetics. Some previous investigators who observed knee kinetic asymmetries in patients following ACL injuries also found asymmetries in GRFs (Decker et al., 2002; Orishimo et al., 2010). Therefore, knee kinetic asymmetries might be predicted by GRF asymmetries. However, no study had examined the relationships between knee kinetic asymmetries and GRF asymmetries during athletic tasks in patients following ACL reconstruction.

Sagittal plane knee kinetic asymmetries have been identified as a risk factor for ACL re-injury (Paterno et al., 2010). Clinically applicable tools are needed to identify the asymmetry and guide the rehabilitation process. The purpose of the current study was to examine the relationships between sagittal plane knee kinetic asymmetries and GRF asymmetries during stop-jump and side-cutting tasks in adolescent patient following ACL-R. It was hypothesized that sagittal plane knee kinetic asymmetries would be correlated with and predicted from GRF asymmetries.

\section{Materials and methods}

Subjects

The current study was a correlational study with one cohort. Twenty-three adolescent patients (gender: 9 males, 14 females; age: $16.5 \pm 1.3$ year; mass: $72.2 \pm 15.6 \mathrm{~kg}$; height: $1.7 \pm 0.1 \mathrm{~m}$ ) participated in the current study. There were 3 contact ACL injuries and 20 non-contact ACL injuries. The time between ACL injury and ACL-R was $1.4 \pm 0.7$ months, with a range of $0.3-3.1$ months. The time between ACL-R and testing was $6.2 \pm 0.6$ months, with a range of 5-7.6 months. The patients were tested around 6 months following surgery as this time point is often used as a rehabilitation milestone for return to sports (Kvist, 2004). All the patients were high school or collegiate athletes who intended to return to sports that involved jump landing and cutting. All the patients received hamstring grafts for ACL-R with a tibial tunnelindependent reconstruction technique (Abebe et al., 2009). Eleven patients injured the dominant leg. Dominant leg was defined as the preferred leg to kick a soccer ball for a further distance. Fifteen patients had concomitant meniscus repair or meniscectomy. All patients had no history of other significant lower extremity injuries. All patients completed standard post-operative physical therapy following the surgery. The study was approved by the Duke Medical Center's Institutional Review Board and all subjects signed informed consent prior to study enrollment.

\section{Data collection}

Subjects first donned spandex and standardized running shoes (Air Pegasus, Nike, Inc., Beaverton, Oregon, USA) for data collection. After the subjects donned the laboratory attire, a 5-min warm-up run was performed on a treadmill at a self-selected running pace. After the warm-up, 46 retro-reflective markers were attached to the subject's bony landmarks (Fig. 1). The coordinate data of the markers were captured using an 8-camera system that was set at a sampling frequency of $120 \mathrm{~Hz}$ (Motion Analysis Corporation, Santa Rosa, California, USA). Following a static standing trial, subjects performed five trials of a vertical stop-jump task and five trials of a left and five trials of a right side-cutting task. The vertical stop-jump task consisted of an approaching run at a maximal speed that terminated in a 2-footed landing on a force plate, which was immediately followed by a 2 -footed takeoff for

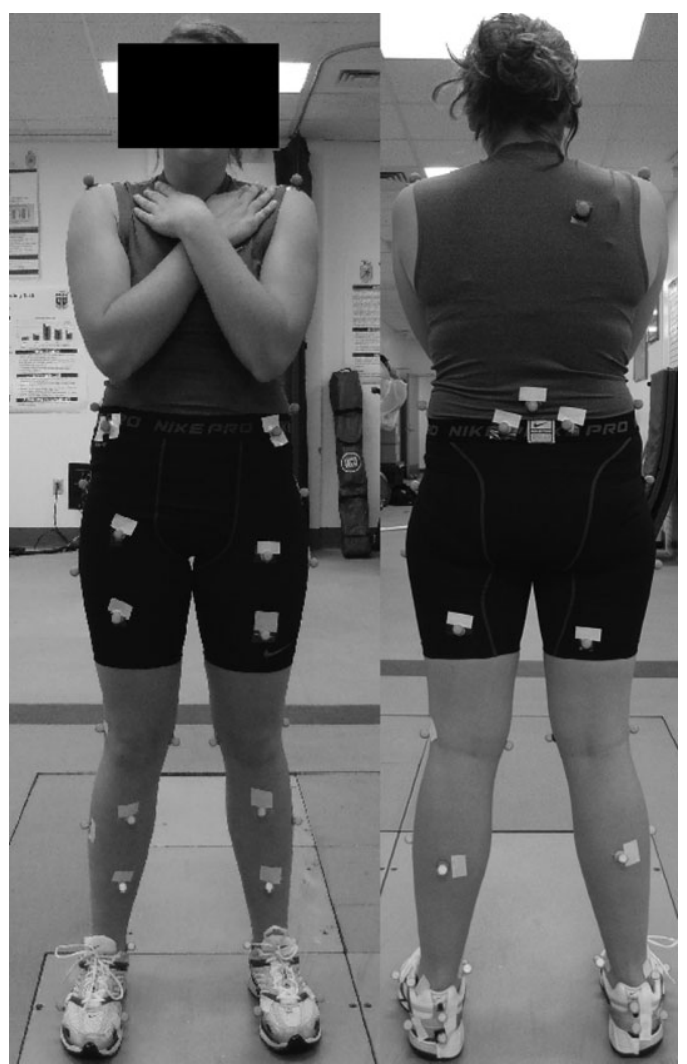

Fig. 1. Front and back views of marker placement.

maximum vertical jump height. The side-cutting task consisted of an approaching run at maximum speed followed by a 1 -footed landing and a subsequent 35 degree cutting maneuver away from the plant foot. Adhesive tape was placed on the ground 35 degree from the running direction as a visual target for the cutting direction. During the stop-jump task, bilateral GRFs were collected during the 2-footed landing phase using two embedded force plates at a sampling rate of 2400 frames/s (AMTI, Watertown, Massachusetts, USA). During the side-cutting task, the GRFs were measured during the 1 -footed landing phase of the cutting maneuver. The subjects conducted the side-cutting task with both the surgical and the non-surgical limbs. The subjects performed the stop-jump task first and then the side-cutting task. The order of cutting direction (cutting toward left or right) for the side-cutting task was randomized.

\section{Data reduction}

The three-dimensional (3D) coordinate data were filtered at $12 \mathrm{~Hz}$, and the GRF data were filtered at $100 \mathrm{~Hz}$ using a low-pass Butterworth filter. The data reduction of sagittal plane knee moments and knee work was performed in Visual 3D (C-Motion, Bethesda, Maryland, USA). Knee joint angular velocities were calculated between thigh and shank reference frames. An inverse dynamic calculation was performed to calculate internal knee joint resultant moments. Knee joint power was calculated as the product of knee joint angular velocity and joint resultant moments. Knee joint work was calculated as the time integration of knee joint power. GRFs were normalized to subject's body weight (N). Joint moments and joint work were normalized to subject's body weight multiplied by body height (m).

For the stop-jump task, the data were analyzed for the 2-footed stance phase. For the side-cutting task, the data were analyzed for the 1-footed stance phase. To assess the knee kinetic asymmetries, 


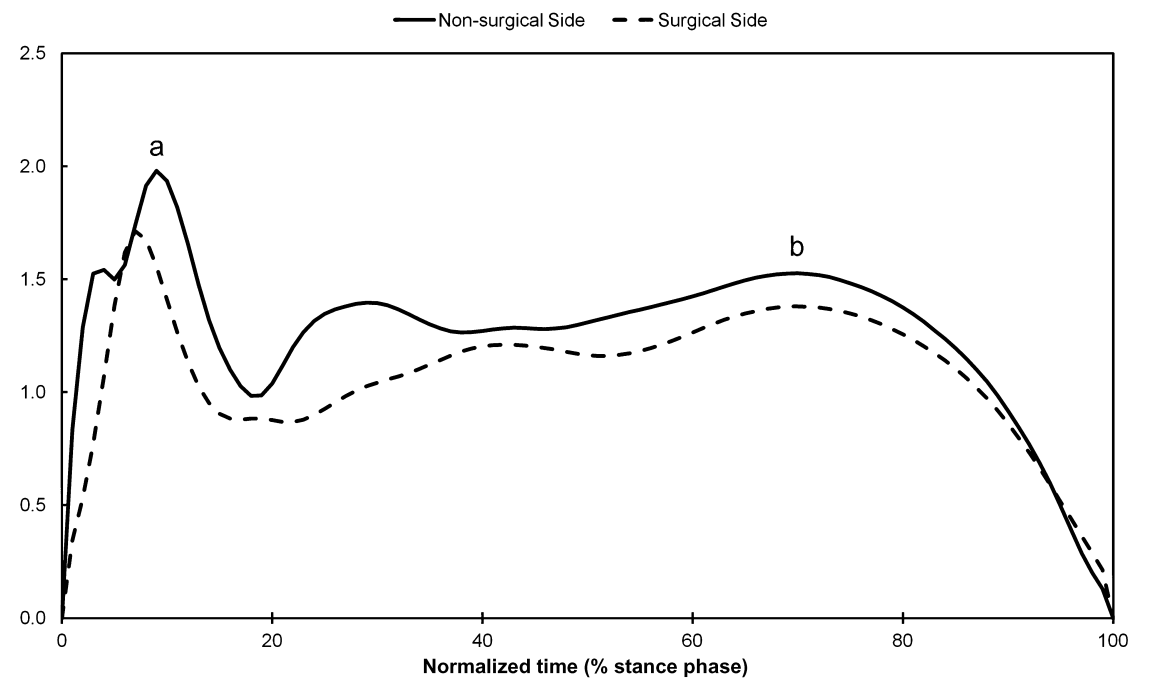

Fig. 2. Representative bilateral vertical ground reaction force (GRF) during stop-jump task. (a) Peak impact vertical GRF. (b) Peak propulsion vertical GRF.

Table 1. Knee kinetic and ground reaction force (GRF) descriptive data (mean \pm SD)

\begin{tabular}{|c|c|c|c|c|}
\hline & \multicolumn{2}{|l|}{ Stop-jump } & \multicolumn{2}{|l|}{ Side-cutting } \\
\hline & Surgical side & Non-surgical side & Surgical side & Non-surgical side \\
\hline Peak knee extension moment (Nm/BW/BH) & $0.104 \pm 0.032$ & $0.142 \pm 0.030$ & $0.127 \pm 0.043$ & $0.168 \pm 0.034$ \\
\hline Average knee extension moment (Nm/BW/BH) & $0.059 \pm 0.021$ & $0.083 \pm 0.015$ & $0.058 \pm 0.022$ & $0.078 \pm 0.017$ \\
\hline Total knee work (J/BW/BH) & $0.095 \pm 0.037$ & $0.141 \pm 0.033$ & $0.055 \pm 0.025$ & $0.086 \pm 0.027$ \\
\hline Vertical GRF impulse (N.s/BW) & $0.348 \pm 0.063$ & $0.431 \pm 0.070$ & $0.364 \pm 0.041$ & $0.402 \pm 0.048$ \\
\hline Anterior-posterior GRF impulse (N-s/BW) & $0.093 \pm 0.030$ & $0.121 \pm 0.024$ & $0.072 \pm 0.018$ & $0.085 \pm 0.026$ \\
\hline Peak impact vertical GRF (N/BW) & $1.61 \pm 0.479$ & $2.02 \pm 0.561$ & $2.60 \pm 0.509$ & $2.82 \pm 0.595$ \\
\hline Peak propulsion vertical GRF (N/BW) & $1.23 \pm 0.196$ & $1.41 \pm 0.197$ & $2.14 \pm 0.211$ & $2.27 \pm 0.134$ \\
\hline Peak posterior GRF (N/BW) & $0.704 \pm 0.312$ & $0.839 \pm 0.263$ & $0.897 \pm 0.279$ & $0.932 \pm 0.344$ \\
\hline Loading rate (N/s/BW) & $44.1 \pm 21.5$ & $57.2 \pm 29.0$ & $65.0 \pm 25.2$ & $73.6 \pm 30.3$ \\
\hline
\end{tabular}

BW, body weight; BH, body height; GRF, ground reaction force.

asymmetry indices were calculated for peak knee extension moment, average knee extension moment, and total knee work during the entire stance phase. Total knee work was the sum of absolute values of both positive and negative work. To assess the GRF asymmetries, asymmetry indices were calculated for vertical GRF impulse during the entire stance phase, anterior-posterior GRF impulse during the entire stance phase, peak impact vertical GRF (Fig. 2), peak propulsion vertical GRF (Fig. 2), peak posterior GRF, and loading rate from initial contact to peak impact vertical GRF. Peak impact vertical GRF was identified as the peak vertical force during early landing. Peak propulsion vertical GRF was identified as peak vertical force around or after middle stance phase. Peak posterior GRF was the peak posterior GRF during the entire stance phase. Peak posterior GRF occurred during early landing phase. All values were calculated and extracted from the time-series data for each task. Asymmetry index was calculated as (non-surgical side-surgical side)/non-surgical side $\times 100 \%$.

\section{Statistical analysis}

Asymmetry indices were averaged across trials for each condition in statistical analysis. To evaluate the relationships between the asymmetries in knee kinetics and GRFs, Pearson's correlation analyses were conducted between each knee kinetic asymmetry index and GRF asymmetry index for the stop-jump and the sidecutting tasks with $r>0.5$ considered "strong", $0.5>r>0.3$ con- sidered "moderate", and $r<0.3$ considered "weak" (Cohen, 1988). To assess the feasibility of predicting knee kinetic asymmetries from GRF asymmetries, step-wise multivariate linear regressions with zero intercept were performed to determine which GRF asymmetry indices could predict the knee kinetic asymmetry indices. The $P$ value for entering or removing a variable in the step-wise regressions was set at 0.05 . Mean absolute error (MAE, eqn. [1]) was used to evaluate how close the predictions were to the actual outcomes. A type I error rate was established at 0.05 for statistical significance. Statistical analyses were conducted in SPSS 12.0.1 (SPSS Inc., Chicago, Illinois USA).

$$
\text { Mean absolute error }=\frac{1}{n} \sum_{i=1}^{n}\left|f_{i}-y_{i}\right|
$$

where $f_{i}$ is the predicted value and $y_{i}$ is the true value.

\section{Results}

The descriptive data for each variable and limb were presented in Table 1. Subjects demonstrated 24-34\% asymmetries in the peak knee extension moment, average knee moment, and total knee work in both stopjump and side-cutting tasks (Table 2). The subjects also had 11-23\% asymmetries in GRFs during the stop-jump 


\section{Dai et al.}

task, while the GRF asymmetries were generally $<11 \%$ in the side-cutting task (Table 2).

For the stop-jump task, Pearson's correlation analysis showed that the asymmetry index for peak knee moments

Table 2. Knee kinetic and ground reaction force (GRF) asymmetry indices (mean \pm SD [min, max])

\begin{tabular}{lcc}
\hline & Stop-jump & Side-cutting \\
\hline Peak knee extension moment (\%) & $26.1 \pm 20.7$ & $24.2 \pm 21.2$ \\
& $(-12.3,68.9)$ & $(-5.7,61.5)$ \\
Average knee extension moment (\%) & $28.4 \pm 22.7$ & $24.4 \pm 24.2$ \\
& $(-5.3,80.1)$ & $(-17.1,73.1)$ \\
Total knee work (\%) & $29.5 \pm 32.1$ & $34.3 \pm 26.1$ \\
& $(-49.5,79.9)$ & $(-11.7,84.2)$ \\
Vertical GRF impulse (\%) & $17.7 \pm 17.3$ & $8.8 \pm 9.4$ \\
& $(-13.2,50.8)$ & $(-9.5,37.5)$ \\
Anterior-posterior GRF impulse (\%) & $22.8 \pm 20.6$ & $11.0 \pm 19.8$ \\
& $(-12.1,67.9)$ & $(-29.4,46.7)$ \\
Peak impact vertical GRF (\%) & $19.3 \pm 16.0$ & $6.2 \pm 13.5$ \\
& $(-12.4,48.6)$ & $(-18.0,27.7)$ \\
Peak propulsion vertical GRF (\%) & $11.7 \pm 12.2$ & $5.8 \pm 8.9$ \\
& $(-8.8,34.8)$ & $(-16.7,32.5)$ \\
Peak posterior GRF (\%) & $16.1 \pm 23.6$ & $-1.4 \pm 24.8$ \\
& $(-32.7,59.6)$ & $(-55.8,46.5)$ \\
Loading rate (\%) & $19.3 \pm 22.9$ & $6.8 \pm 23.3$ \\
& $(-24.4,56.5)$ & $(-30.3,58.3)$ \\
\hline
\end{tabular}

were significantly correlated with all GRF asymmetry indices (Table 3). Asymmetry indices for average knee moments and knee work were significantly correlated with all GRF asymmetry indices, except for loading rate (Table 3). The step-wise regressions to predict asymmetry indices for peak knee moments (Table 4, Fig. 3), average knee moments (Table 4, Fig. 4), and knee work (Table 4, Fig. 5), all included the asymmetry index for the vertical GRF impulse as the only predictor. The MAEs of the three prediction equations were 11-14\%.

For the side-cutting task, Pearson's correlation analysis demonstrated that asymmetry indices for peak knee moments, average knee moments, and knee work were only significantly correlated with the vertical GRF impulse and peak propulsion vertical GRF asymmetry indices (Table 3). The step-wise regression to predict peak knee moments included vertical GRF impulse in the first model and both vertical GRF impulse and peak posterior GRF in the second model (Table 4, Fig. 6). The step-wise regressions to predict the average knee moment (Table 4, Fig. 7) and knee work asymmetry indices (Table 4, Fig. 8) both included the vertical GRF impulse asymmetry index as the only predictor. The MAEs of the three prediction equations were 16-19\%.

Table 3. Pearson's correlation coefficients ( $P$ values) between knee kinetic asymmetry indices and ground reaction force (GRF) asymmetry indices

\begin{tabular}{|c|c|c|c|c|c|c|}
\hline & $\begin{array}{l}\text { Vertical } \\
\text { GRF impulse }\end{array}$ & $\begin{array}{l}\text { Anterior-posterior } \\
\text { GRF impulse }\end{array}$ & $\begin{array}{l}\text { Peak impact } \\
\text { vertical GRF }\end{array}$ & $\begin{array}{l}\text { Peak propulsion } \\
\text { vertical GRF }\end{array}$ & $\begin{array}{l}\text { Peak posterior } \\
\text { GRF }\end{array}$ & $\begin{array}{l}\text { Loading } \\
\text { rate }\end{array}$ \\
\hline \multicolumn{7}{|l|}{ Stop-jump } \\
\hline Peak knee extension moment & $\begin{array}{c}0.74 \\
(<0.001)\end{array}$ & $\begin{array}{c}0.57 \\
(<0.01)\end{array}$ & $\begin{array}{c}0.64 \\
(<0.001)\end{array}$ & $\begin{array}{l}0.50 \\
(0.01)\end{array}$ & $\begin{array}{c}0.45 \\
(0.03)\end{array}$ & $\begin{array}{c}0.44 \\
(0.04)\end{array}$ \\
\hline Average knee extension moment & $\begin{array}{c}0.85 \\
(\leq 0.001)\end{array}$ & $\begin{array}{c}0.76 \\
(<0.001)\end{array}$ & $\begin{array}{c}0.61 \\
(<0.01)\end{array}$ & $\begin{array}{c}0.66 \\
(<0.001)\end{array}$ & $\begin{array}{c}0.57 \\
(<0.01)\end{array}$ & $\begin{array}{l}0.29 \\
(0.18)\end{array}$ \\
\hline Total knee work & $\begin{array}{c}0.83 \\
(<0.001)\end{array}$ & $\begin{array}{c}0.72 \\
(<0.001)\end{array}$ & $\begin{array}{c}0.53 \\
(<0.01)\end{array}$ & $\begin{array}{c}0.42 \\
(\underline{0.04})\end{array}$ & $\begin{array}{c}0.65 \\
(<0.001)\end{array}$ & $\begin{array}{c}0.09 \\
(0.69)\end{array}$ \\
\hline \multicolumn{7}{|l|}{ Side-cutting } \\
\hline Peak knee extension moment & $\begin{array}{c}0.46 \\
(\underline{0.03})\end{array}$ & $\begin{array}{c}0.19 \\
(0.40)\end{array}$ & $\begin{array}{c}0.25 \\
(0.25)\end{array}$ & $\begin{array}{c}0.49 \\
(\underline{0.02})\end{array}$ & $\begin{array}{c}0.13 \\
(0.55)\end{array}$ & $\begin{array}{c}0.09 \\
(0.67)\end{array}$ \\
\hline Average knee extension moment & $\begin{array}{c}0.53 \\
(<0.01)\end{array}$ & $\begin{array}{l}0.26 \\
(0.23)\end{array}$ & $\begin{array}{l}0.35 \\
(0.10)\end{array}$ & $\begin{array}{l}0.48 \\
(\underline{0.02})\end{array}$ & $\begin{array}{l}0.23 \\
(0.29)\end{array}$ & $\begin{array}{c}0.20 \\
(0.37)\end{array}$ \\
\hline Total knee work & $\begin{array}{c}0.65 \\
(\leq 0.001)\end{array}$ & $\begin{array}{l}0.41 \\
(0.06)\end{array}$ & $\begin{array}{l}0.41 \\
(0.06)\end{array}$ & $\begin{array}{l}0.41 \\
(\underline{0.05})\end{array}$ & $\begin{array}{l}0.34 \\
(0.11)\end{array}$ & $\begin{array}{c}0.20 \\
(0.35)\end{array}$ \\
\hline
\end{tabular}

Significant correlations were underlined.

Table 4. Regression models to predict knee kinetic asymmetry indices

\begin{tabular}{|c|c|c|c|c|c|}
\hline & Predictor & $\begin{array}{l}\text { Unstandardized regression } \\
\text { coefficients }\end{array}$ & $R^{2}$ & $P$ value & $\begin{array}{l}\text { Mean absolute error } \\
\text { (mean } \pm \text { SD, \%) }\end{array}$ \\
\hline \multicolumn{6}{|l|}{ Stop-jump } \\
\hline Peak knee extension moment & Vertical GRF impulse & 1.19 & 0.78 & $<0.001$ & $11.9 \pm 9.9$ \\
\hline Average knee extension moment & Vertical GRF impulse & 1.37 & 0.86 & $<0.001$ & $11.4 \pm 7.6$ \\
\hline Total knee work & Vertical GRF impulse & 1.61 & 0.84 & $<0.001$ & $13.7 \pm 11.0$ \\
\hline \multicolumn{6}{|l|}{ Side-cutting } \\
\hline \multirow[t]{2}{*}{ Peak knee extension moment } & Model I Vertical GRF impulse & 1.86 & 0.55 & $<0.001$ & $16.4 \pm 14.0$ \\
\hline & $\begin{array}{ll}\text { Model II } & \text { Vertical GRF impulse } \\
& \text { and peak posterior GRF }\end{array}$ & $\begin{array}{l}\text { Vertical GRF impulse: } 2.22 \text {; } \\
\text { Peak posterior GRF: }-0.44\end{array}$ & 0.65 & $<0.001$ & $14.4 \pm 12.7$ \\
\hline Average knee extension moment & Vertical GRF impulse & 2.03 & 0.58 & $<0.001$ & $17 \pm 14.1$ \\
\hline Total knee work & Vertical GRF impulse & 2.80 & 0.70 & $<0.001$ & $18.7 \pm 14.5$ \\
\hline
\end{tabular}

GRF, ground reaction force. 


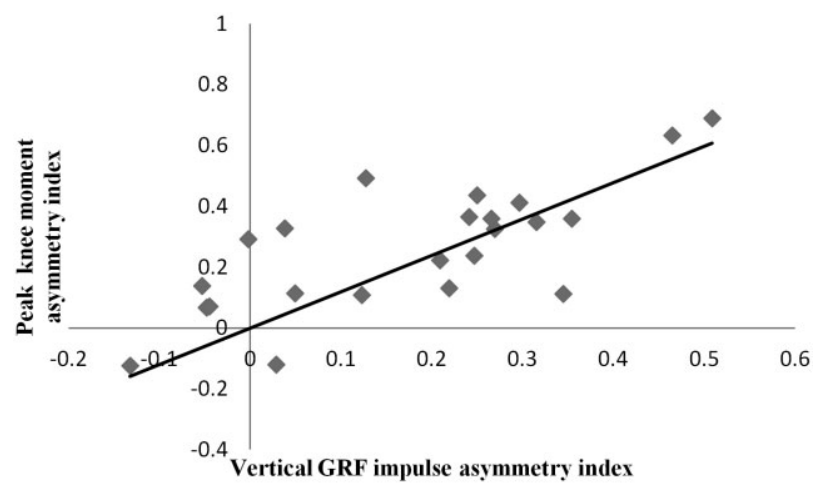

Fig. 3. Peak knee moment asymmetry index as a function of vertical ground reaction force (GRF) impulse asymmetry index during stop-jump.

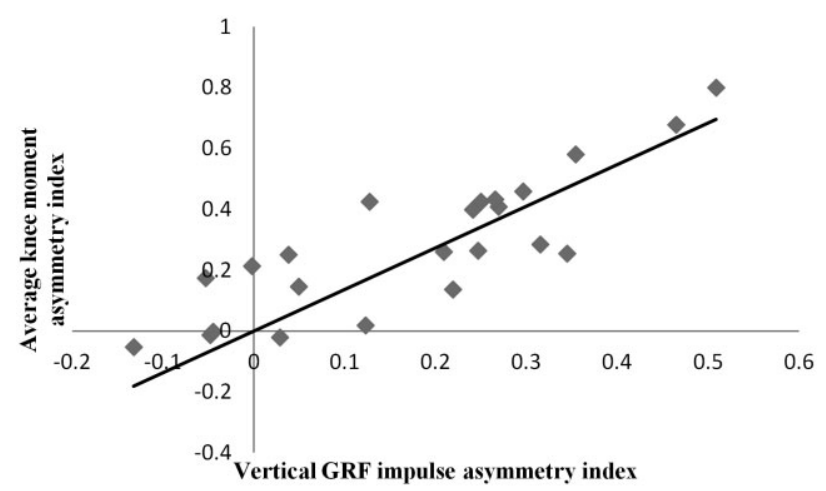

Fig. 4. Average knee moment asymmetry index as a function of vertical ground reaction force (GRF) impulse asymmetry index during stop-jump.

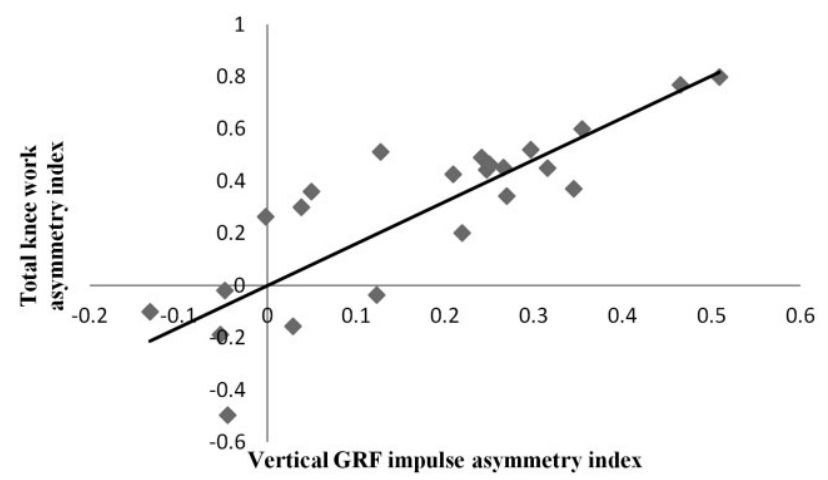

Fig. 5. Knee work asymmetry index as a function of vertical ground reaction force (GRF) impulse asymmetry index during stop-jump.

\section{Discussion}

Sagittal plane knee moment asymmetry during landing has been reported as a risk factor for ACL re-injury (Paterno et al., 2010). Unfortunately, knee kinetic asymmetry following ACL reconstruction is commonly observed (Decker et al., 2002; Orishimo et al., 2010). Consistent with previous studies, the current study has found more than a $24 \%$ asymmetry in knee moments and knee work between the surgical and non-surgical limb

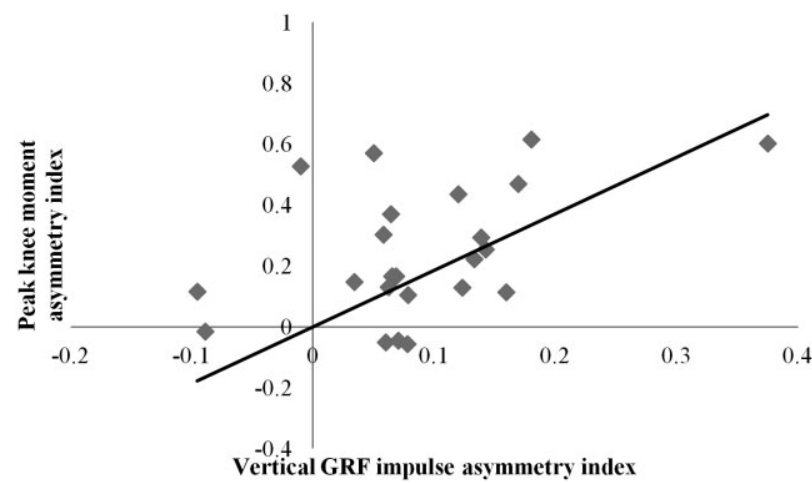

Fig. 6. Peak knee moment asymmetry index as a function of vertical ground reaction force (GRF) impulse asymmetry index during side-cutting.

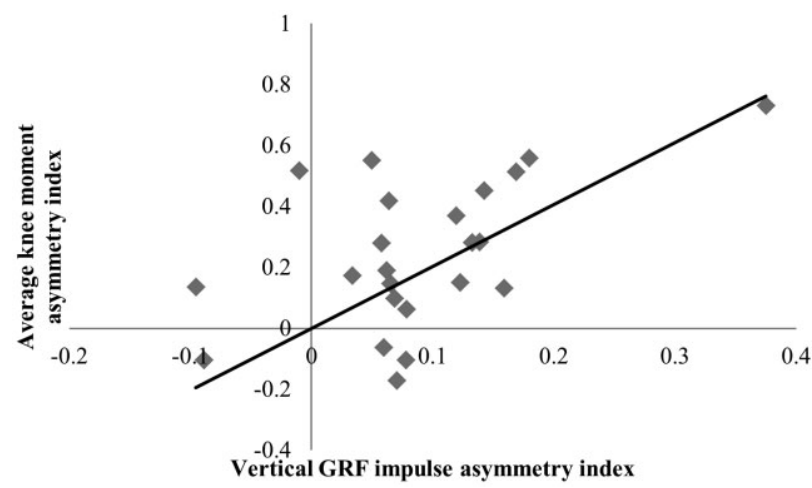

Fig. 7. Average knee moment asymmetry index as a function of vertical ground reaction force (GRF) impulse asymmetry index during side-cutting.

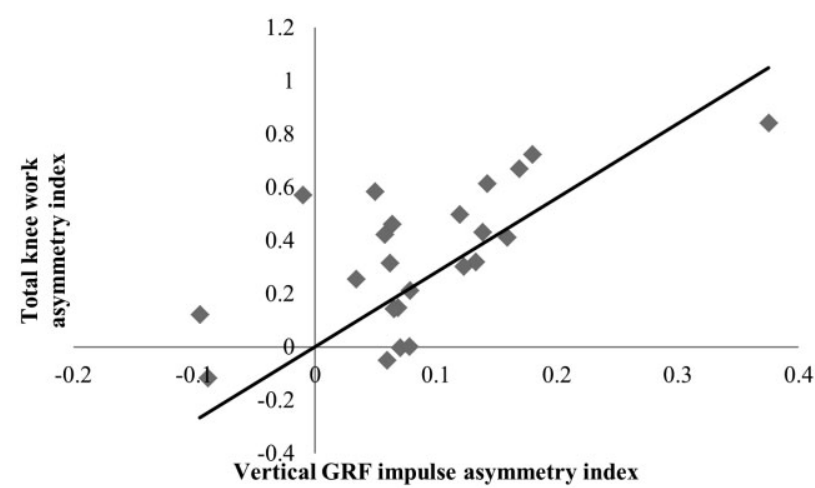

Fig. 8. Knee work asymmetry index as a function of vertical ground reaction force (GRF) impulse asymmetry index during side-cutting.

during both stop-jump and side-cutting tasks. The significant knee kinetic asymmetries suggest that modifiable re-injury risk factors may still exist at the time of discharge from rehabilitation. The current study evaluated sagittal plane knee kinetic and GRF asymmetries as well as the relationships between them in order to identify variables that were systematically associated with asymmetry. Commonly used variables including peak knee moments, average knee moments, and knee work 


\section{Dai et al.}

were chosen as the kinetic variables of interest (Decker et al., 2002; Orishimo et al., 2010). GRF variables at specific events as well as GRF impulse were chosen as the GRF variables of interest.

Using the inverse dynamics, knee moments are determined by the magnitude of the external GRFs, the GRF moment arms to the knee joint center, and the product terms of angular acceleration and moment of inertia. The increased asymmetries at the knee joint compared to GRF asymmetries suggest that the knee kinetic asymmetries were not only caused by the asymmetries in the magnitude of external GRFs but also in other components such as the moment arms of GRFs. The GRF moment arms are affected to some extent by the amount of knee flexion. Increased knee flexion usually increases the GRF moment arm to the knee joint center. Previous investigators have demonstrated that the surgical knee usually has decreased knee flexion when compared to the non-surgical knee during landing in ACL injured patients (Gokeler et al., 2010; Orishimo et al., 2010). The decreased knee flexion angle could contribute to the increased asymmetries at the knee joint. However, this speculation might not be true if the magnitude of the GRF increased at the same time when knee flexion decreased. The decreased GRF was a mechanical representation of decreased knee moment. The inherent factors that caused the decreased knee moment on the surgical side could be deficits in dynamic strength, muscle inhibition, altered neuromuscular function, or fear of re-injury (Ingersoll et al., 2008; Ardern et al., 2011).

The hypothesis of the current study was supported by significantly moderate to strong correlations between the sagittal plane knee kinetic and certain GRF asymmetries. Because GRFs are determinant components in calculating knee kinetics, it was not surprising to observe significant correlations between GRFs and knee kinetics asymmetries. However, it should be noted that not all GRF asymmetries were correlated with knee kinetics asymmetries. The knee kinetic asymmetries were associated with most asymmetries in GRFs during the stop-jump task. The stronger correlations between knee kinetic asymmetries and GRF asymmetries during the stop-jump than during the side-cutting could be due to the nature of the double-leg support task. A double-leg support task allows subjects to use inter-limb compensation strategies that have the potential to increase limb asymmetries. To achieve the performance goal of stopping and jumping for a maximum height, subjects generally utilized the non-surgical side more than the surgical side during both landing and jumping. Previous investigators have observed a higher contralateral ACL injury rate than ACL graft injury rate in adolescent and young patients who sustained ACL injuries (Shelbourne et al., 2009b; Paterno et al., 2010). The abnormal interlimb compensation with more loads on the contralateral side might contribute to the high contralateral ACL injury rate. In contrast to the stop-jump task, the subjects were constrained to use the surgical side only to complete the side-cutting task. Subjects are only able to use inter-joint compensation during a single-leg landing task. Knee kinetic asymmetries were only significantly correlated with asymmetries in peak propulsion GRF and vertical GRF impulse. Considering the landing phase involves both passive and active tissues to absorb the impact force, it was reasonable that the impairments in knee function were more likely to correlate with the GRF during the push-off phase, which involves mainly active muscle contractions.

In all regression models aimed at predicting the knee kinetic asymmetries, vertical GRF impulse asymmetry was included as the predictor. Only the second regression model to predict peak knee moment asymmetry during side-cutting included both vertical GRF impulse and peak posterior GRF. Considering a lack of significant relationship between asymmetry indices for peak posterior GRF and peak knee moment and a negative coefficient for asymmetry index for peak posterior GRF in the second model, the second model might not be more logical than the first model. Although knee kinetic asymmetries were associated with asymmetries in many GRF parameters, asymmetries in the vertical GRF impulse was the most sensitive variable that was able to explain the majority of the variance in the knee kinetic asymmetries. In the regression models, the coefficients for the vertical GRF impulse were larger than 1, which suggested that small asymmetries in vertical GRF impulse were associated with large asymmetries in knee kinetics. Compared to peak impact GRF and peak propulsion GRF that were commonly used discrete variables (Decker et al., 2002; Paterno et al., 2007, 2011), vertical GRF impulse represented the vertical GRF during the entire stance phase and might give a better representation of overall function.

The findings indicate that a force plate or sensor with a single vertical axis (Neitzel et al., 2002) may be able to achieve the goal of identifying knee kinetic asymmetries, which increases the potential clinical application of this assessment. Compared to an inverse dynamics, simply measuring vertical GRF does not require high-speed cameras and sophisticated calculations. In addition, using portable force plates/sensor increases the flexibility of testing locations. This may provide a clinical model for assessing patients' knee kinetic asymmetries during the course of rehabilitation as well as understanding how specific interventions affect kinetic asymmetries. It should be noted that the prediction errors were approximately $12 \%$ for stop-jump task and $17 \%$ for sidecutting task. The regression equation was able to predict the changes in knee kinetic asymmetries as a function of vertical GRF impulse, but the errors should be considered when the exact value of the asymmetries are estimated. The use of 3D motion analysis should still be considered a more accurate and sensitive assessment of 
ACL injuries and ACL re-injuries; however, this method would improve testing feasibility in larger populations and allow for a more accessible test that could be completed in clinical settings.

There were several limitations of the current study. GRF asymmetries predicted knee kinetic asymmetries, which could predict ACL re-injury. However, it was unknown if GRF asymmetries could prospectively predict ACL re-injuries. A self-reported knee health quality of life measure could better describe the subjects but was not included in the study. Subjects were tested approximately 6 months after ACL-R due to this being a standard return to athletics time point. However, not all patients had been released to return to sports at the time of testing. No control group was included. However, the asymmetry indices observed in the current study were greater than the asymmetry indices in normal control groups in previous literature (van der Harst et al., 2007; Paterno et al., 2011). Only adolescent patients were included in the current study because of their high ACL re-injury rate. Future studies need to include adult populations to better understand the broader application of this method of data collection. Patients were tested in athletic tasks, which may or may not correlate with the testing of movement patterns at a lower level of loading. Future studies need to study tasks that are commonly used during rehabilitation and the effect that training interventions have on these variables. A leave-one-out test was not performed to evaluate the predictive power of GRF asymmetries because of a relatively small sample size. Some markers were placed on spandex and footwear, which might introduce error in tracking motion of body segments. The current study provides a clinically applicable model for the potential identification of the knee kinetic asymmetries, which has previously been associated with an elevated risk of re-injury. Additional work is necessary to determine clinically relevant cut-off values for asymmetries in vertical GRF impulse that will allow for appropriate progression during rehabilitation and eventual return to play decisions.

\section{Perspectives}

Sagittal plane kinetic asymmetries were risk factors for ACL re-injuries. Sagittal plane knee kinetic asymmetries were associated with GRF asymmetries during a stopjump task and a side-cutting task in adolescent patients following ACL-R. Asymmetries in the vertical GRF impulse significantly predicted knee kinetic asymmetries with 11-19\% estimation errors during both tasks. A single-axis force transducer with low cost and flexible testing locations may have the ability to monitor sagittal plane knee kinetic asymmetries during the rehabilitation progress, which would be expected to assist in optimizing patient outcomes and minimizing re-injury rates.

Key words: ACL injury, biomechanics, jump, cutting, landing.

\section{Acknowledgements}

We thank DonJoy Orthopaedics for the research support that was received for this project. We also thank the K-lab staff for their help with data collection.

\section{References}

Abebe ES, Moorman CT, Dziedzic TS, Spritzer CE, Cothran RL, Taylor DC, Garrett WE, DeFrate LE. Femoral tunnel placement during anterior cruciate ligament reconstruction: an in vivo imaging analysis comparing transtibial and 2-incision tibial tunnel-independent techniques. Am J Sports Med 2009: 37 (10): 1904-1911.

Ardern CL, Webster KE, Taylor NF, Feller JA. Return to sport following anterior cruciate ligament reconstruction surgery: a systematic review and meta-analysis of the state of play. Br J Sports Med 2011: 45 (7): 596-606.

Barber-Westin SD, Noyes FR. Factors used to determine return to unrestricted sports activities after anterior cruciate ligament reconstruction. Arthroscopy 2011: 27 (12): 1697-1705.

Boden BP, Dean GS, Feagin JA, Garrett WE. Mechanisms of anterior cruciate ligament injury. Orthopedics 2000: 23 (6): 573-578.
Cohen J. Statistical power analysis for the behavioural sciences. 2nd edn. Hillsdale, NJ: Lawrence Erlbaum Associates, 1988.

Decker MJ, Torry MR, Noonan TJ, Riviere A, Sterett WI. Landing adaptations after ACL reconstruction. Med Sci Sports Exerc 2002: 34 (9): 1408-1413.

Fink C, Hoser C, Hackl W, Navarro RA, Benedetto KP. Long-term outcome of operative or nonoperative treatment of anterior cruciate ligament rupture - is sports activity a determining variable? Int J Sports Med 2001: 22 (4): 304-309.

Gokeler A, Hof AL, Arnold MP, Dijkstra PU, Postema K, Otten E. Abnormal landing strategies after ACL reconstruction. Scand J Med Sci Sports 2010: 20 (1): e12-e19.

Granan LP, Bahr R, Steindal K, Furnes O, Engebretsen L. Development of a national cruciate ligament surgery registry: the Norwegian national knee ligament registry. Am J Sports Med 2008: 36 (2): 308-315.

van der Harst JJ, Gokeler A, Hof AL. Leg kinematics and kinetics in landing from a single-leg hop for distance. A comparison between dominant and non-dominant leg. Clin Biomech 2007: 22 (6): 674-680.

Ingersoll CD, Grindstaff TL, Pietrosimone BG, Hart JM. Neuromuscular consequences of anterior cruciate ligament injury. Clin Sports Med 2008: 27 (3): 383-404, vii.

Kvist J. Rehabilitation following anterior cruciate ligament injury: current recommendations for sports participation. Sports Med 2004: 34 (4): 269-280.

Miyasaka KC, Daniel DM, Stone ML, Hirshman P. The incidence of knee ligament injuries in the general population. Am J Knee Surg 1991: 4: 3-8.

Myer GD, Ford KR, Khoury J, Succop P, Hewett TE. Development and 


\section{Dai et al.}

validation of a clinic-based prediction tool to identify female athletes at high risk for anterior cruciate ligament injury. Am J Sports Med 2010: 38 (10): 2025-2033.

Neitzel JA, Kernozek TW, Davies GJ. Loading response following anterior cruciate ligament reconstruction during the parallel squat exercise. Clin Biomech 2002: 17 (7): 551-554.

Orishimo KF, Kremenic IJ, Mullaney MJ, McHugh MP, Nicholas SJ. Adaptations in single-leg hop biomechanics following anterior cruciate ligament reconstruction. Knee Surg Sports Traumatol Arthrosc 2010: 18 (11): 1587-1593.

Paterno MV, Ford KR, Myer GD, Heyl R, Hewett TE. Limb asymmetries in landing and jumping 2 years following anterior cruciate ligament reconstruction. Clin J Sport Med 2007: 17 (4): 258-262.

Paterno MV, Schmitt LC, Ford KR, Rauh MJ, Myer GD, Hewett TE. Effects of sex on compensatory landing strategies upon return to sport after anterior cruciate ligament reconstruction. J Orthop Sports Phys Ther 2011: 41 (8): 553-559.

Paterno MV, Schmitt LC, Ford KR, Rauh MJ, Myer GD, Huang B, Hewett TE. Biomechanical measures during landing and postural stability predict second anterior cruciate ligament injury after anterior cruciate ligament reconstruction and return to sport. Am J Sports Med 2010: 38 (10): 1968-1978.
Salmon L, Russell V, Musgrove T, Pinczewski L, Refshauge K. Incidence and risk factors for graft rupture and contralateral rupture after anterior cruciate ligament reconstruction. Arthroscopy 2005: 21 (8): 948-957.

Shelbourne KD, Gray T, Haro M. Incidence of subsequent injury to either knee within 5 years after anterior cruciate ligament reconstruction with patellar tendon autograft. Am J Sports Med 2009a: 37 (2): 246-251.

Shelbourne KD, Sullivan AN, Bohard K, Gray T, Urch SE. Return to basketball and soccer after anterior cruciate ligament reconstruction in competitive school-aged athletes. Sports Health 2009b: 1 (3): 236-241. 